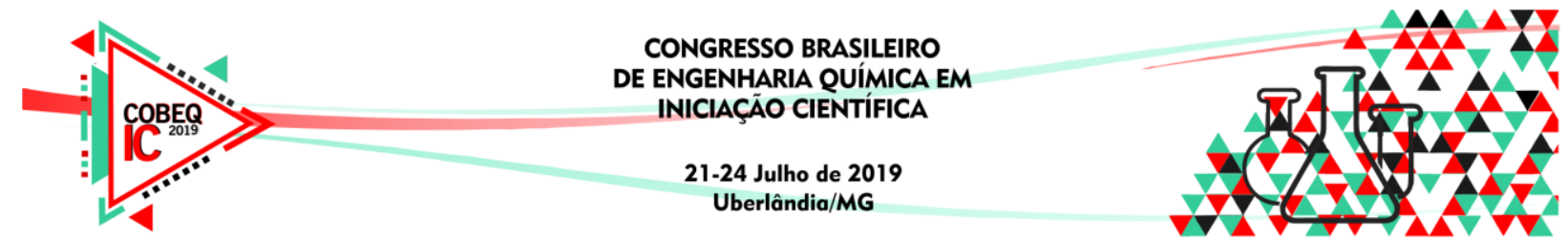

\title{
DESENVOLVIMENTO DE KITS EXPERIMENTAIS DE BAIXO CUSTO UTILIZANDO PLATAFORMA LIVRE PARA O ENSINO E PESQUISA DE CONTROLE DE PROCESSOS
}

\author{
B. C. M. HENRIQUE ${ }^{1}$, M. A. VILELA ${ }^{2}$, L. C. M. HENRIQUE ${ }^{3}$ e H. M. HENRIQUE $^{2}$ \\ ${ }^{1}$ Universidade Federal de Uberlândia, Faculdade de Engenharia Elétrica \\ ${ }^{2}$ Universidade Federal de Uberlândia, Faculdade de Engenharia Química \\ ${ }^{3}$ Universidade Federal de Uberlândia, Faculdade de Engenharia Mecânica \\ E-mail para contato: humberto@ufu.br
}

\begin{abstract}
RESUMO - O presente trabalho apresenta a implementação de dois kits experimentais para laboratórios de controle e automação utilizando hardware e softwares livres e de baixo custo. A plataforma Visual Studio Code (livre) foi utilizada para desenvolver os códigos fontes e interfaces dos kits e a plataforma de prototipagem eletrônica open source Arduino (livre) foi utilizada para desenvolver o hardware das montagens experimentais. Além de abordar as questões teóricas e conceituais do Arduino, o presente trabalho apresenta descritivos das montagens com foco no desenvolvimento de controladores reais. Os resultados demonstraram que a plataforma desenvolvida se mostrou adequada para o uso em kits experimentais permitindo ao usuário confrontar suas ideias e expectativas com as evidências experimentais. Mostrou-se ainda que a instrumentação é de fácil configuração, com baixo nível de ruído e custo baixo, o que torna viável a construção de kits desta natureza para fins didáticos e de pesquisa.
\end{abstract}

\section{INTRODUÇÃO}

O ensino e pesquisa de controle de processos na academia está baseada em demasia em pacotes de simulação disponíveis e em laboratórios virtuais, ambos com sua posição insubstituível no processo de ensino e pesquisa. Mas, infelizmente, os experimentos com malhas de controle são muitas vezes limitados a esses domínios virtuais e os alunos não têm o feedback do mundo físico e real sobre o impacto dos algoritmos de controle e seus parâmetros. As razões para isso residem no fato do elevado custo do hardware e software necessários para implementar algoritmos de controle fisicamente e em tempo real. Este trabalho tem como objetivo desenvolver uma plataforma experimental de baixo custo, direta e surpreendentemente poderosa para a implementação de algoritmos de controle em tempo real. A plataforma consiste em placas Arduino e um microcomputador de baixo custo rodando sob plataforma Windows ${ }^{\mathrm{TM}}$ para interfacear com os sensores de baixo custo compatíveis com a plataforma Arduino e com a planta experimental real. A placa Arduino é usada para interação com o mundo físico através de suas entradas e saídas. Os algoritmos de controle foram implementados usando o software open-source Visual Studio Code, que permitem que alunos desenvolvam seus algoritmos de controle sem custo adicional. O ensino e a pesquisa em controle e automação em cursos de graduação e pós-graduação no Brasil são muito caros por envolver o uso de hardware e softwares proprietários de elevado custo. Por causa disso, 


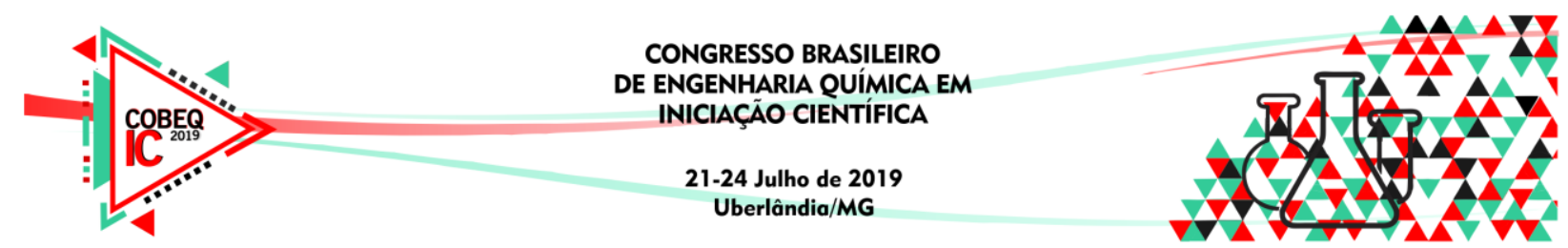

cursos de controle e automação são relegados à abordagem teórica, deixando o aluno sem a vivência prática dessas tecnologias. $\mathrm{O}$ trabalho tem por objetivo desenvolver kits experimentais de baixo custo para uso em laboratórios de automação e controle de processos para que possam ser usados em atividades de ensino e pesquisa nas disciplinas associadas ao tema.

\section{A PLATAFORMA DESENVOLVIDA}

O microcomputador no qual a plataforma foi baseada forma par perfeito para a educação e pesquisa de controle e automação, combinando o poder computacional da plataforma VSCode e a capacidade de entrada-saída das placas Arduino. Parte integrante da plataforma apresentada é o software que transforma essas placas em um controlador industrial programável, pois permite que os alunos/usuários criem algoritmos de controle usando as mesmas ferramentas de software e conceitos de fluxo de trabalho que são usados no desenvolvimento de algoritmos de controladores industriais. A plataforma de controle desenvolvida consistiu de placa microcontroladora Arduino UNO R3, um microcomputador Dell $8^{\text {a }}$ Geração Intel Core i5 8GB RAM 500 GB com Windows 10 e o pacote Visual Studio Code que é um editor de código-fonte desenvolvido pela Microsoft para Windows, Linux e macOS. Os componentes individuais são discutidos nas próximas seções.

\subsection{A placa Arduino}

O Arduino (disponível em: <https://code.visualstudio.com/>, 2019) é uma plataforma de prototipagem eletrônica de código aberto bem conhecida que consiste de uma unidade de controle central de muitas aplicações de controle incorporadas, desde roupas interativas até robótica e impressoras 3D. No presente trabalho a placa foi programada para atuar como escravo (slave) e suas entradas e saídas foram usadas para interação com o mundo físico (Figura 1a). Neste trabalho usou-se as placas Arduino UNO R3 para a unidade de controle de vazão e para a unidade de controle de nível. Esta placa traz em seu núcleo funções para leitura de sinais analógicos através da utilização do conversor analógico digital. A placa Arduino UNO possui pinos de entrada e saídas digitais, assim como pinos de entradas e saídas analógicas. Essa placa possui 14 pinos que podem ser usados como entradas ou saídas digitais (Figura 1b). Alguns desse pinos podem ser usados como saídas PWM de 8 bit. A placa possui ainda 6 entradas com resolução de 10 bits. $\mathrm{O}$ clock do conversor AD do Atmega328 permite a aquisição de 15400 amostras por segundo.

Figura 1 - A plataforma de ensino e pesquisa Arduino/Visual Studio Code. (a). Esquema de ligação. (b). Detalhes da Arduino UNO R3.
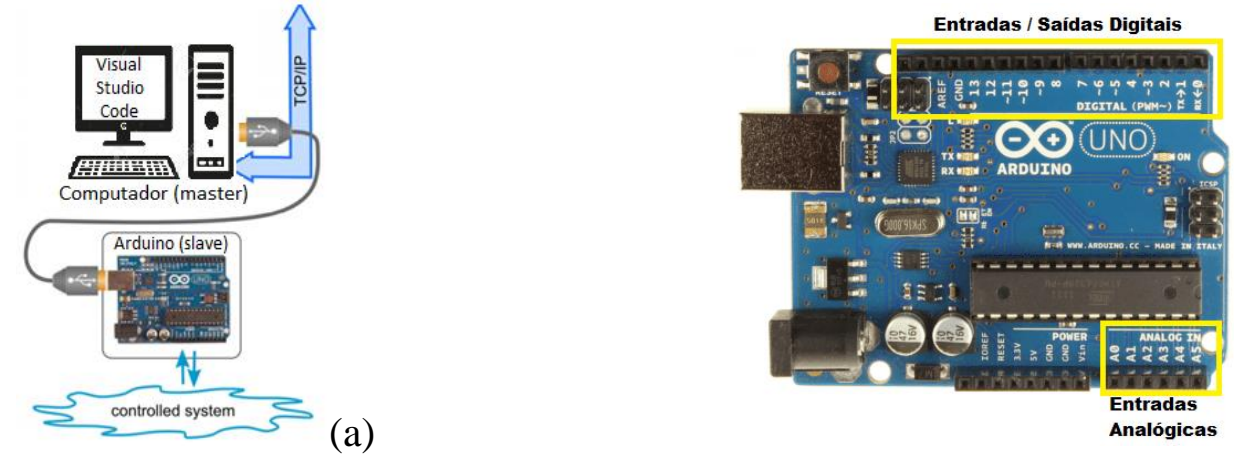


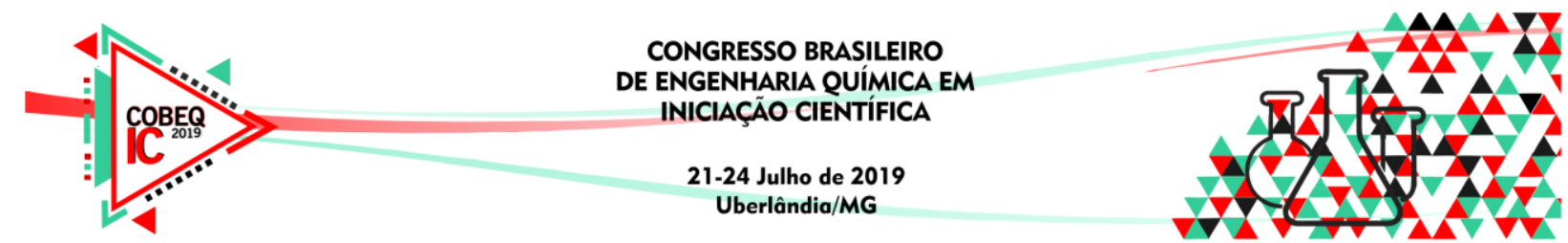

\subsection{O Visual Studio Code (VSCode)}

O VSCode (disponível em: < https://code.visualstudio.com/>, 2019) é um programa gratuito de código aberto desenvolvido pela Microsoft com suporte para Windows, macOS e Linux. É altamente customizável, permitindo que os usuários mudem o tema do editor, as teclas de atalho, entre outros aspectos. Ele é mais vantajoso em relação à IDE do Arduino principalmente nos aspectos gráfico e de depuração de erros (debugging). O VSCode possui uma extensão chamada PlatformIO IDE que é um sistema de código aberto para desenvolvimento em IoT (Internet of Things). É escrito em Python e não necessita de nenhuma biblioteca ou ferramenta adicional. Adicionalmente, o editor possui suporte à sintaxe de diversas linguagens como Python, Ruby, C, $\mathrm{C}^{++}$, Visual Basic, C Sharp e F Sharp.

\subsection{Controle em tempo real}

A última etapa da criação de um sistema de controle em tempo real foi a implantação do algoritmo de controle na plataforma de destino. Os algoritmos de controle tipo PID (Seborg, 2004) foram implementados em linguagem $\mathrm{C}^{++}$dentro do VSCode com saídas gráficas e exportação dos dados referentes às variáveis controladas, manipuladas e setpoints. $\mathrm{O}$ protocolo de comunicação usado entre o master e o slave foi o padrão serial utilizando um cabo USB em modo assíncrono com baud rate de 115200 bps, 8 bits de dados sem paridade e 1 bit de parada. O programa já implementando a estratégia de controle foi então compilado pelo compilador $\mathrm{C}^{++}$do VSCode e foi carregado na memória flash do microcontrolador Arduino via cabo USB.

\section{AS PLANTAS EXPERIMENTAIS}

Duas plantas experimentais de bancada foram construídas e instrumentadas com sensores de baixo custo. A primeira planta é de controle de vazão e a segunda de nível. As plantas são detalhadas a seguir.

\subsection{Sistema de controle de vazão}

O primeiro sistema experimental construído consta de um pequeno tanque de um litro de capacidade, de onde é bombeado água por um sensor de vazão de efeito Hall (modelo YFS402 de 0,5-5,0 L/min). Este sensor gera como saída um sinal de tensão na forma de uma onda quadrada de 0 a $5 \mathrm{~V}$ com frequência variável em função da vazão medida. Este sinal, que é um sinal digital, é então adquirido por um dos canais digitais da Arduino UNO e o período da onda é medido usando-se a função pulseIn() de biblioteca do VSCode. Esse sensor foi previamente calibrado usando uma balança e cronômetro. Duas minibombas centrífugas (modelo RS-385 DC 12V e 0-2 L/min) em paralelo são utilizadas para impulsionar o líquido por um sensor de vazão. A vazão das bombas é manipulada alterando a tensão nos terminais da bomba de 0 a $12 \mathrm{~V}$ utilizando de um motor shield para Arduino (modelo L293D Driver Ponte H) que controla até 4 motores DC. Este shield recebe sinal PWM da placa Arduino. Dois pinos PWM do Arduino UNO são, então, usados para manipular a vazão das bombas utilizando a função de biblioteca analogWrite() do VSCode. O líquido bombeado fica em circuito fechado evitando o desperdício de água durante o funcionamento da unidade experimental. A Figura 2a mostra uma foto da unidade. 


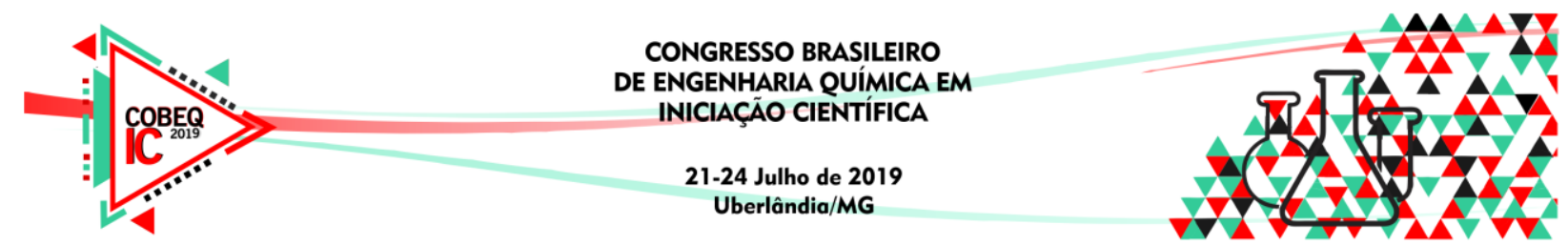

Figura 2 - Montagens experimentais com detalhes da instrumentação. (a). Controle de vazão. (b). Controle de nível.
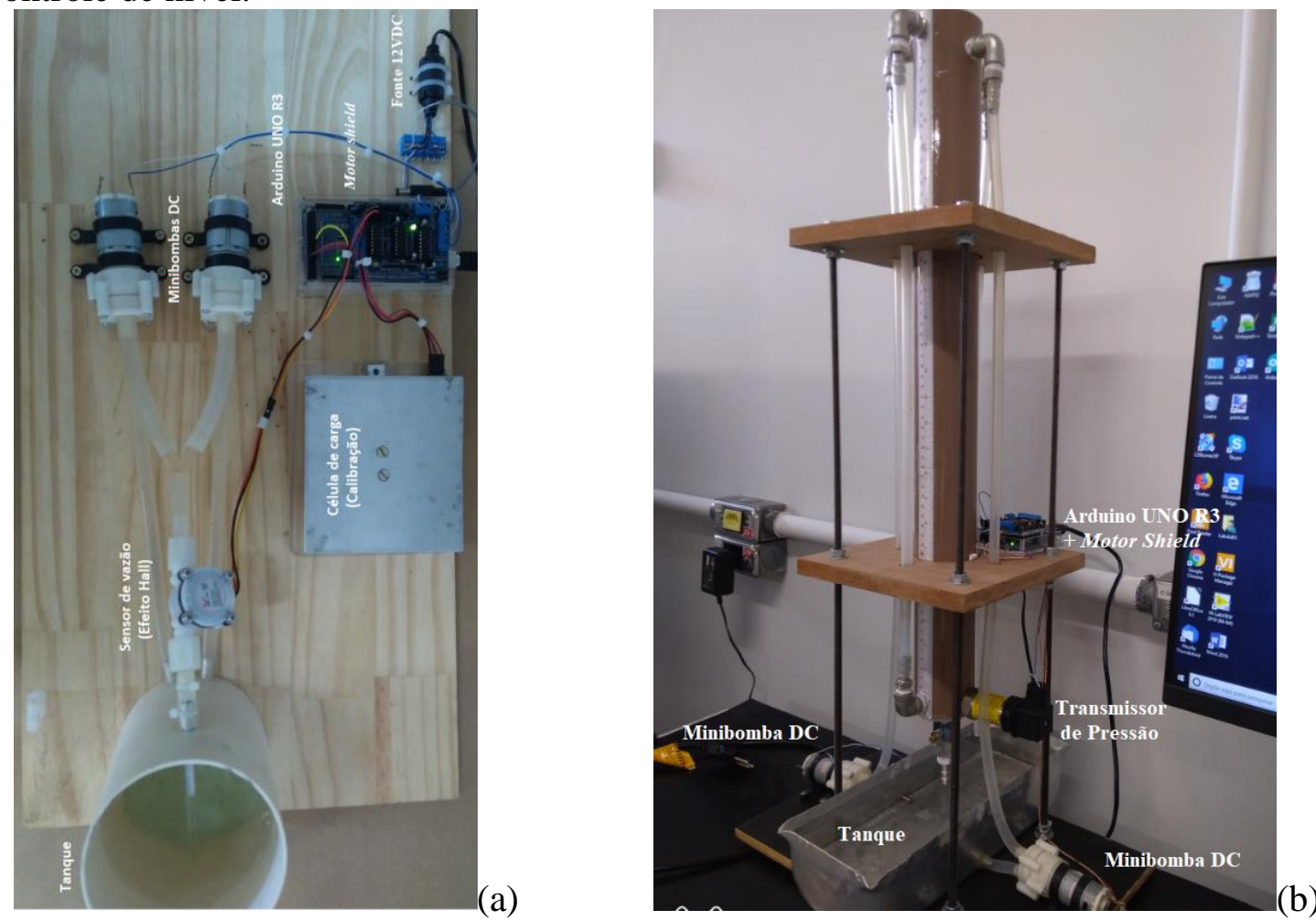

\subsection{Sistema de controle de nível}

O segundo sistema é um tanque cilíndrico de PVC de $65 \mathrm{~cm}$ de altura e 50,8 $\mathrm{mm}$ de diâmetro, que é alimentado no topo por duas correntes de líquido provenientes de duas minibombas. A vazão das bombas é manipulada alterando a tensão nos terminais das mesmas de 0 a 12 V utilizando um motor shield (modelo L293D Driver Ponte H). Uma válvula manual tipo esfera de $1 / 4$ " foi instalada no fundo do tanque. Na base do tanque foi instalado um transmissor de pressão diferencial (modelo JF302 piezoresistivo $10 \mathrm{kPa} / 0.1$ bar gauge). $\mathrm{O}$ sinal de saída deste transmissor é um sinal de 4-20 mA que é enviado para um conversor corrente/tensão (4-20ma para 0-5v), uma vez que a placa Arduino UNO somente recebe sinal analógico de tensão na faixa de $0-5 \mathrm{~V}$. Assim, é possível adquirir um sinal analógico em um dos pinos de entrada do Arduino UNO que é proporcional ao nível de líquido dentro do tanque. A Figura $2 b$ mostra uma foto da unidade.

\section{RESULTADOS}

\subsection{Identificação dos sistemas}

Nas montagens construídas as entradas do sistema dinâmico são sinais com resolução de 8 bits (0-255) que geram sinais PWM com duty cicle de 0 a 100\% em pinos específicos para saídas PWM na placa Arduino UNO R3. Os sinais PWM são, então, convertidos pelo motor shield em sinais analógicos de tensão de 0 a 12VDC que alimentam as minibombas DC. Dessa forma as vazões das minibombas são manipuladas. As saídas são a vazão medida pelo sensor de efeito Hall na primeira montagem e o nível medido pelo transmissor de pressão 

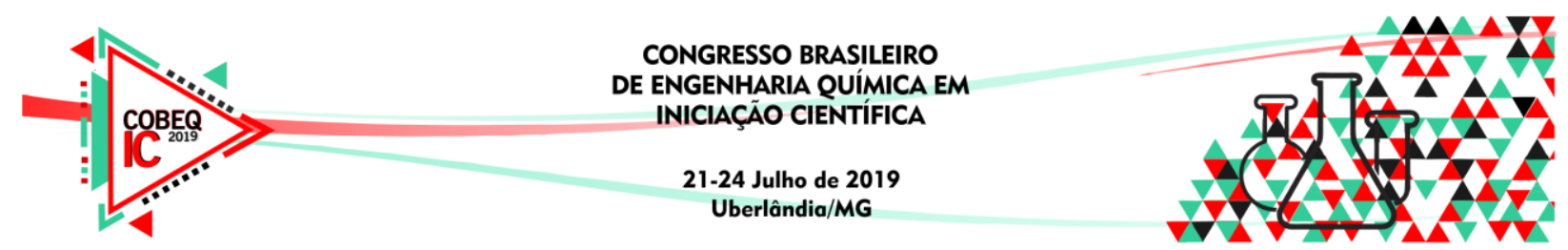

diferencial na segunda montagem, ambos devida e previamente calibrados. Para obtenção das funções de transferência dos sistemas uma corrida em malha aberta foi executada variando-se os sinais enviados aos pinos PWM entre os valores de 90 a 255, correspondendo às vazões mínima e máxima obtidas nas minibombas. Os dados dessas corridas foram aqui omitidos por falta de espaço. Com os dados entrada versus saída de cada planta em mãos, funções de transferência tipo first order plus time delay (FOPDT) foram numericamente ajustadas aos dados (Equações (1) e (2)):

$$
\begin{aligned}
& Q^{\prime}(s)=\frac{K}{\tau s+1} U^{\prime}(s) \\
& \left(K=8,57 \mathrm{~cm}^{3} \mathrm{~min}^{-1} \text { e } \tau=2,92 \mathrm{~s}\right) \\
& H^{\prime}(s)=\frac{K_{1}}{\tau_{1} s+1} e^{-\theta_{1} s} U_{1}(s)+\frac{K_{2}}{\tau_{2} s+1} e^{-\theta_{2} s} U_{2}(s) \\
& \left(K_{1}=0,378 \mathrm{~cm} ; \tau_{1}=44,76 \mathrm{~s} ; K_{2}=0,414 \mathrm{~cm}, \tau_{2}=47,10 \mathrm{~s} \mathrm{e} \theta_{1}=\theta_{2}=10 \mathrm{~s}\right)
\end{aligned}
$$

\subsection{Resposta em malha fechada}

De posse das funções de transferências ajustou-se controladores tipo PI para ambas as plantas usando o método IMC (Rivera et. al., 1986) para a planta de vazão e o método ITAE Load e Setpoint (Seborg et. al., 2004) para a planta de nível. A Tabela 1 mostra os parâmetros ajustados para os controladores. Para testar o desempenho real do controlador de vazão foi introduzida uma sequência de modificações no setpoint da vazão. Os resultados são mostrados na Figura 3a, onde observa-se a performance bastante adequada do controlador PI, sem introduzir oscilações e sem offset na variável controlada. Já a Figura 3 b revela a performance de um controlador $\mathrm{P}$, utilizando o mesmo $\mathrm{K}_{\mathrm{C}}$ do controlador PI, porém, com $\tau_{\mathrm{I}}$ infinito. Observa-se com clareza a presença de offset conforme prevê a teoria. Observa-se também dessas Figuras que as ações de controle (variável manipulada) sofreram grandes variações no momento da introdução dos degraus no setpoint. Essas grandes variações podem ser suavizadas ajustando o controlador de maneira mais conservadora. Contudo, haverá uma degradação na performance da malha fechada.

Figura 3 - Desempenho experimental da malha de controle de vazão frente a mudanças no setpoint. (a). Controlador PI. (b). Controlador P.
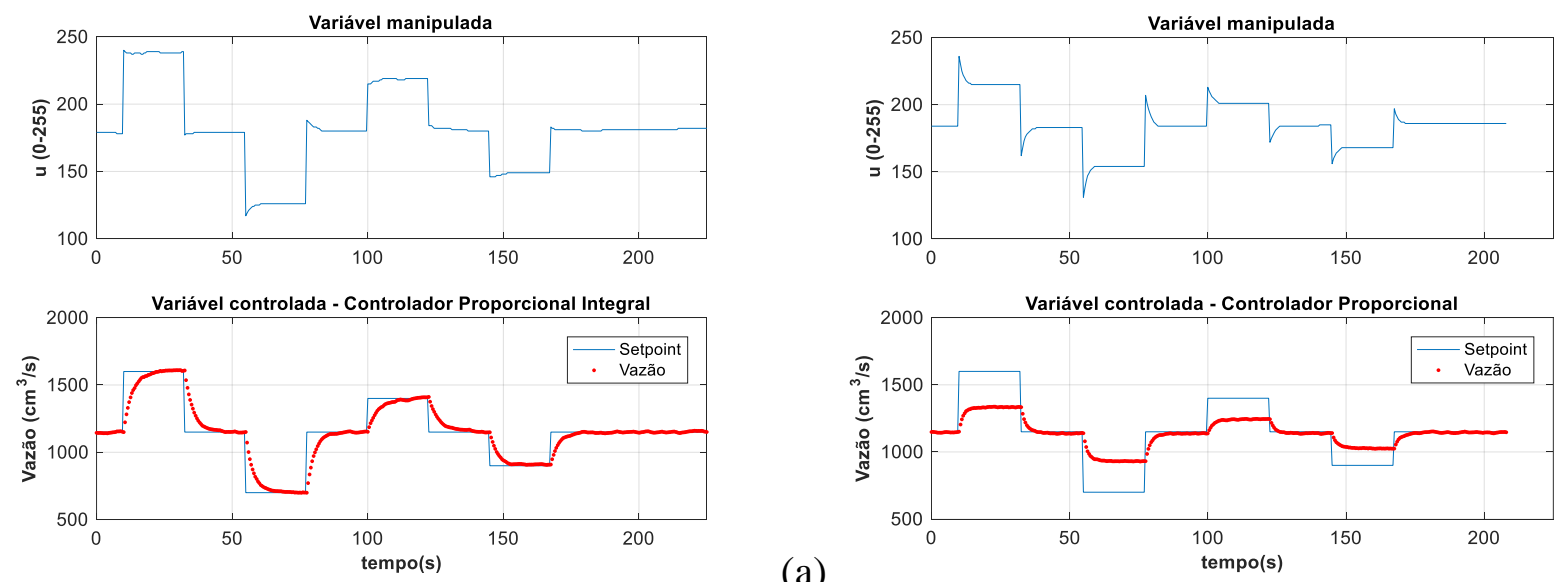

(a) 


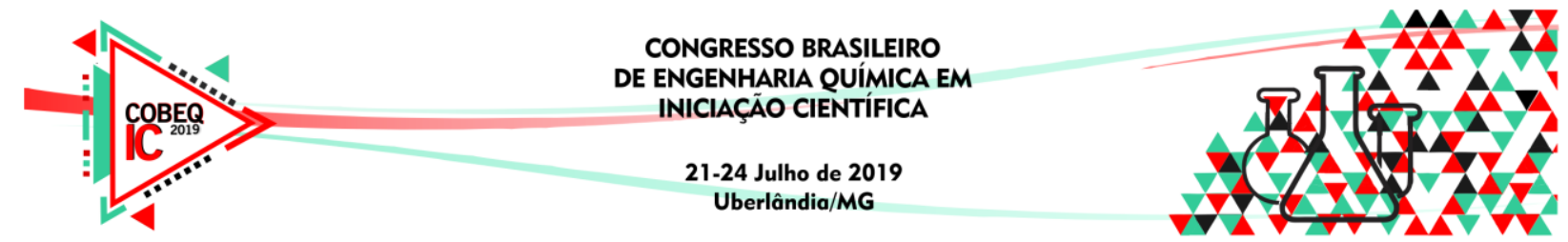

Figura 4 - Desempenho experimental da malha de controle de nível frente a mudanças no: (a). Setpoint e controlador PISetpoint. (b). Carga e controlador PILoad.
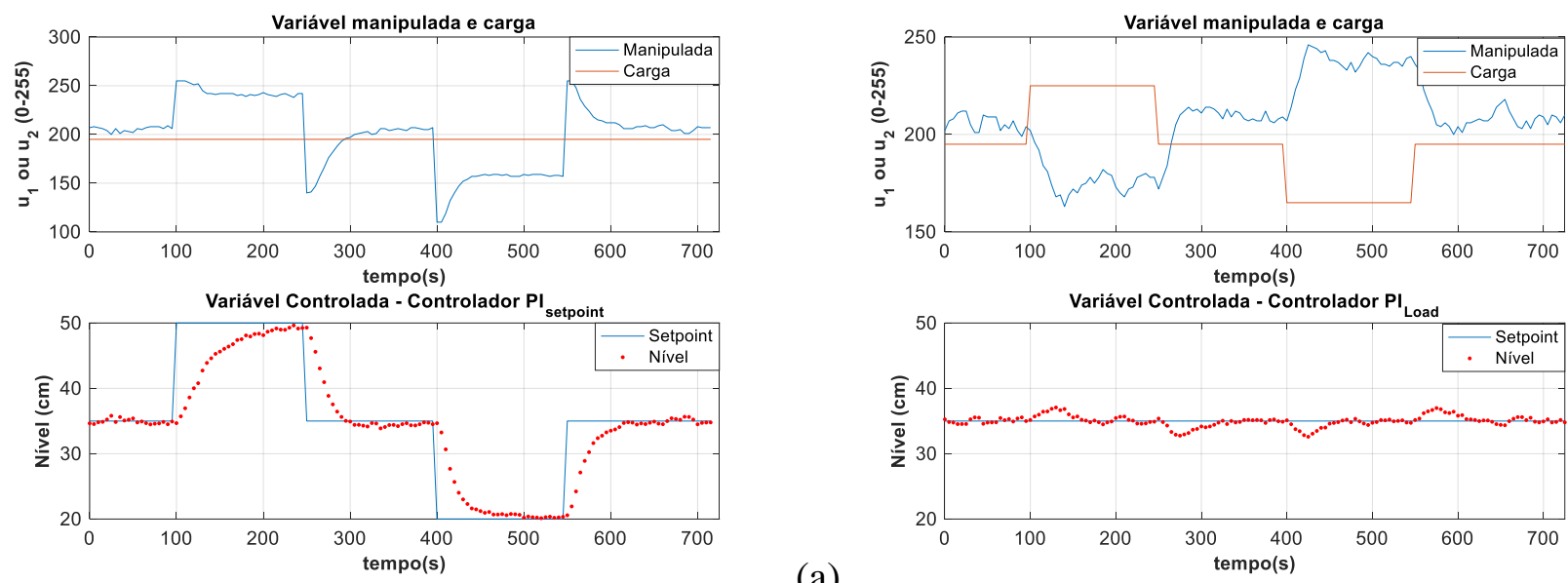

(a)

Tabela 1 - Parâmetros de sintonia dos controladores PI desenvolvidos.

\begin{tabular}{lccc}
\hline \multicolumn{1}{c}{ Problema } & Método & $\mathrm{K}_{\mathrm{C}}$ & $\tau_{\mathrm{I}}$ \\
\hline Vazão (setpoint) & IMC & $0,117 \mathrm{~cm}^{-3} \cdot \min$ & $2,92 \mathrm{~s}$ \\
Nível (setpoint) & ITAE setpoint & $6,124 \mathrm{~cm}^{-1}$ & $45,07 \mathrm{~s}$ \\
Nível (Load) & ITAE Load & $9,428 \mathrm{~cm}^{-1}$ & $24,36 \mathrm{~s}$ \\
\hline
\end{tabular}

Neste caso também foi introduzida uma sequência de modificações no setpoint. Os resultados são mostrados na Figura 4a, onde observa-se a performance bastante adequada do controlador PISetpoint, sem introduzir oscilações e sem offset na variável controlada. Já a Figura 4b mostra os resultados do controlador $\mathrm{PI}_{\text {Load }}$ frente a perturbações na carga. Observa-se que o controlador conseguiu rejeitar as perturbações na carga sem degradar a performance da variável controlada, isto é, sem oscilações e sem offset.

\subsection{Custo da Instrumentação das Unidades Experimentais}

A Tabela 2 e 3 traz os custos associados com a instrumentação das unidades. Chama muito a atenção o baixo custo dos instrumentos e da placa Arduino UNO.

Tabela 2 - Custo da instrumentação da unidade de controle de vazão.

\begin{tabular}{|c|c|c|c|c|}
\hline Item & Descrição & Quan & Isto (Us\$)* & Total \\
\hline Minibomba & Modelo RS-385 DC 9 a $15 \mathrm{~V}$ e $0-2 \mathrm{~L} / \mathrm{min}$ & 02 & 11,29 & 22,58 \\
\hline Sensor Hall & $\begin{array}{l}\text { Medidor de vazão modelo YF S402 de efeito } \\
\text { Hall e } 0,5-5,0 \mathrm{~L} / \mathrm{min}\end{array}$ & 01 & 9,96 & 9,96 \\
\hline Ponte H 293D & $\begin{array}{l}\text { Motor Shield Arduino L293D Driver Ponte H } \\
\text { Placa com microcontrolador Atmega328, } 14\end{array}$ & 01 & 1,49 & 1,49 \\
\hline Arduino UNO I & $\begin{array}{l}3 \text { entradas/saídas digitais (6 são saídas PWM), } \\
6 \text { entradas analógicas. }\end{array}$ & 01 & 6,50 & 6,5 \\
\hline VSCode & $\begin{array}{l}\text { Programa open-source com suporte para } \\
\text { Windows, macOS e Linux. }\end{array}$ & 01 & 0,0 & 0,0 \\
\hline
\end{tabular}

*. Custo FOB, não inclui frete até o destino.

Total $=$ Us $\$ 40,53$ 


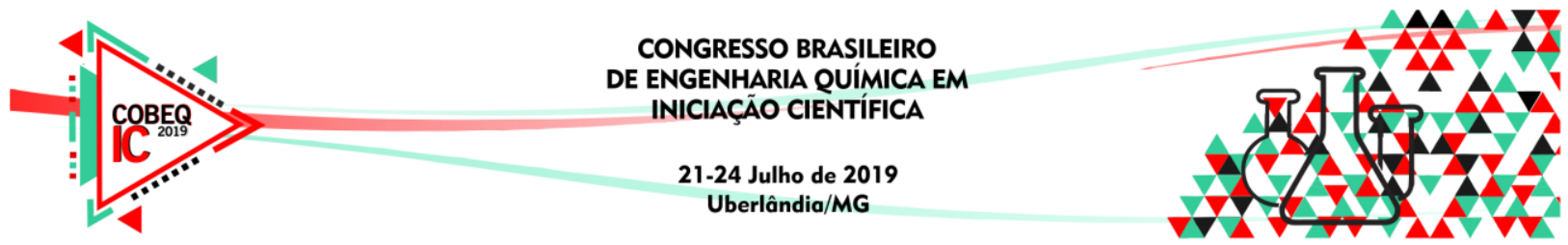

Tabela 3 - Custo da instrumentação da unidade de controle de nível.

\begin{tabular}{|c|c|c|c|c|}
\hline Item & Descrição & Quant & sto (Us\$)* & Total \\
\hline Minibomba & Modelo RS-385 DC 9 a $15 \mathrm{~V}$ e $0-2 \mathrm{~L} / \mathrm{min}$ & 02 & 11,29 & 22,58 \\
\hline $\begin{array}{l}\text { Sensor pressão } \\
\text { diferencial }\end{array}$ & $\begin{array}{l}\text { Modelo JF302 de } 4-20 \mathrm{~mA}, 12-36 \mathrm{~V} \text { de } \\
\text { 10kpa/0.1bar gauge e aço inoxidável } 316 .\end{array}$ & 01 & 32,00 & 32,00 \\
\hline Conversor $4-20 \mathrm{~mA}$ & Modulo Conversor 4-20ma para $0-5 \mathrm{~V}$. & 01 & 12,86 & 12,86 \\
\hline Ponte H 293D & $\begin{array}{l}\text { Motor Shield Arduino L293D Driver H } \\
\text { Placa com microcontrolador Atmega328, }\end{array}$ & 01 & 1,49 & 1,49 \\
\hline Arduino UNO R3 & $\begin{array}{l}14 \text { entradas/saídas digitais ( } 6 \text { são saídas } \\
\text { PWM), } 6 \text { entradas analógicas. }\end{array}$ & 01 & 6,50 & 6,5 \\
\hline VSCode & $\begin{array}{l}\text { Programa open-source com suporte para } \\
\text { Windows, macOS e Linux. }\end{array}$ & 01 & 0,0 & 0,0 \\
\hline
\end{tabular}

*. Custo FOB, não inclui frete até o destino.

Total $=\mathbf{U s} \$ 75,43$

\section{CONCLUSÃO}

Os resultados demonstraram que a plataforma Arduino juntamente com o software VSCode se mostram eficientes para o uso em kits experimentais de controle de processos. A instrumentação é de fácil configuração, com baixo nível de ruído e custo baixo, o que torna viável a construção de kits desta natureza para fins didáticos e de pesquisa em ambiente universitário.

Do ponto de vista pedagógico, a vantagem dessa abordagem de ensino e pesquisa é que o aluno confronta as suas ideias e expectativas com as evidências experimentais, tornando-se gradualmente competente no processo de coordenação das teorias de controle e automação com as evidências experimentais que produz. Assim, essa plataforma de ensino e pesquisa renova os papéis dos alunos e dos professores envolvidos com o ensino e a pesquisa de controle e automação de processos.

\section{REFERÊNCIAS}

, Microsoft Visual Studio Code, 2019. Disponível em: < https://code.visualstudio.com/>. Acesso em 15 de abril de 2019.

Arduino, 2019. Disponível em: <https://www.arduino.cc/>. Acesso em 15 de abril de 2019.

Rivera, D. E.; Morari, M.; Skogestad, S. Internal model control: PID controller design. Industrial \& Engineering Chemistry Process Design and Development,1986 25 (1), p. 252265

Seborg, D. E.; Edgar, T. F.; Mellichamp, D. A. Process Dynamics and Control, Wiley, New York, 2004. 